\title{
HOW TO SECURELY BROADCAST A SECRET
}

\author{
Jörg Schwenk \\ Deutsche Telekom AG, Technologiezentrum, Am Kavalleriesand 3, D-64295 Darmstadt - GER- \\ MANY \\ schwenk@tzd.telekom.de
}

\begin{abstract}
The conference key distribution problem over a broadcast channel, i.e. distribution of a key to a set of recipients by a central authority, can be solved by true broadcasting using threshold schemes. This paper gives a security analysis of this approach. After describing succesful attacks on two special implementations of true broadcasting schemes, a slightly different scheme is shown to be provably secure under some well-known cryptographic assumptions. As an application of the threshold scheme approach, a subliminal channel free scheme is given which can be used e.g. in Pay-TV systems.
\end{abstract}

Keywords: Conference key distribution, one-way function, chosen plaintext attack, subliminal channel, broadcast channel

\section{INTRODUCTION}

A single central authority, the transmitter, wishes to broadcast a secret $s$ selectively only to a subset of the set of all users. The users which belong to this subset are the recipients, all other users are non-recipients. The nonrecipients should not be able to learn anything about the secret. Each time the transmitter wishes to broadcast a new secret (e.g. a session key for a conference or for a pay-TV event), the set of recipients may have changed completely. To be able to distinguish the variable subset of recipients from the non-recipients, each user $U_{i}$ must be assigned a personal key $k_{i}$ which is only known to him and the transmitter.

One obvious possibility to achieve this is to encrypt the secret $s$ under each of the recipients personal keys and to broadcast the ciphertexts. Each recipient will then have to filter out the ciphertext belonging to his personal key and decrypt it to get the secret $s$.

The intention of the scheme proposed by Berkovits in [Ber91] is to establish a new cryptographic primitive called true broadcasting to selectively broadcast a secret without using encryption. This is done by broadcasting shares of the 
secret $s$ obtained by using Threshold schemes (see Definition 2). Unfortunately it is not clear if the general scheme given in [Ber91] is secure since the two implementations of the scheme can be broken completely in the sense of Definition 1. In [JKKO95], some new implemetations of true broadcasting are given using techniques from number theory.

However, by using only temporary shares which are derived in a probabilistic way from the real shares, the system can be made provably secure against all types of attacks. The basic idea goes back to a publication of Laih and Yen [LY93]. We are able to give complete security proofs for the broadcasting schemes which are based on this idea.

Definition 1 (1) A broadcast scheme is secure if any set of (polynomially bounded) adversaries that is disjoint to the set of recipients is not able to compute the secret.

(2) A broadcast scheme is completely broken if some group of users can compute the secret personal key of another user.

Our scheme does not compete with the broadcast encryption schemes introduced by Fiat and Naor in [FN93]. In their model the (large) group of recipients only changes slightly from round to round so that it makes sense to ignore the plaintext message describing the set of recipients. Our scheme may in contrast be used for (small) groups of recipients which change completely from round to round. Please note that Definition 1 does not pose any restrictions on the size of the set of adversaries. This should be compared with broadcast encryption schemes [FN93], where security is only guaranteed for sets up to a certain cardinality $k$.

One-time broadcast encryption schemes have been studied in a number of papers, e.g. [Sti97, SvT98]. These schemes are, as their name indicates, provably secure in an information theoretic sense if they are only used once. In contrast our scheme is only computationally secure (because we use one-way functions), but can be used a polynomial number of times in the length of the input (e.g. the length of the pseudoshares). Combinatorial bounds for such schemes have been investigated in [BC94, LS98], using entropy notation in [BC94] and a set theoretic approach in [LS98]. In [BC94], the keys can be reused a fixed number of times.

One additional advantage of avoiding the use of encryption techniques are regulatory aspects. When a sequence of ciphertexts is broadcast, a governmental authority can never be sure about the contents of the ciphertexts unless it has decrypted all of them. In our scheme, the authority could be given two additional shares so that it could compute the secret with the help of one share, verify its contents, and verify the consistency of the complete system with the second share. The authority can be sure that only one secret is broadcast, and that no subliminal channel is present. 
The main idea of [Ber91] is to use $(k, n)$-Threshold Schemes [Sha79] to broadcast the secret.

Definition 2 In a $(k, n)$-Threshold Scheme a secret $s$ is split into $n$ shares such that the secret can be reconstructed from any $k$ shares, but knowing at most $k-1$ shares does not give any information about the secret in an information theoretic sense.

In Berkovits' scheme, each user $U_{i}$ gets as his personal key a pseudoshare $s_{i}$. To broadcast a secret $s$ to a set of $k$ recipients, the transmitter proceeds as follows:

- Construct a $(k+j+1,2 k+j+1)$-Threshold Scheme $\mathcal{T}$ for $j \geq 0$ such that:

- $s$ is the secret of the Threshold Scheme $\mathcal{T}$.

- The pseudoshares of the $k$ recipients are used as shares in $\mathcal{T}$.

- The pseudoshares of the non-recipients are not used as shares.

- $j$ additional shares are used for the construction of $\mathcal{T}$.

- Broadcast $k+j$ shares of the Threshold Scheme which are different from pseudoshares of the recipients.

Each recipient has to add his own pseudoshare to the $k+j$ broadcast shares to be able to reconstruct the secret $s$. A non-recipient learns nothing about the secret because he knows only $k+j$ shares.

In Section 2. we show how to break two concrete implementations of this general scheme. The idea of Laih and Yen [LY93] is presented in Section 3. The main difference of the scheme presented there in comparison to Berkovits' scheme is that the personal keys of the recipients are not used directly as shares, but only their randomized images under some one-way function. By making some explicit assumptions on the one-way function used, we can prove the security of the new general scheme. Section 4 . shows how to use the scheme to establish a secure key distribution scheme without any subliminal channel. Finally, Section 5. gives a concrete application of the scheme in the area of Pay-TV systems.

\section{ATTACKS ON BERKOVITS SCHEME}

In this section we briefly describe two special implementations of broadcast schemes given in [Ber91] and how they can be broken completely.

The Polynomial Scheme. Each user $U_{i}$ gets as his secret pseudoshare a point $\left(x_{i}, y_{i}\right) \in G F(q)^{2}$. The $x_{i}$ must be pairwise different. To broadcast the 
secret $s$ to the recipients $U_{1}, \ldots, U_{k}$, the transmitter computes a polynomial $p(x)$ of degree $k+j$ through the $k+1$ points $(0, s),\left(x_{1}, y_{1}\right), \ldots,\left(x_{k}, y_{k}\right)$ and through $j \geq 0$ additional random points $\left(r_{1}, t_{1}\right), \ldots,\left(r_{j}, t_{j}\right)$. The transmitter now broadcasts $k+j$ points on $p(x)$ which are different from $\left(x_{i}, y_{i}\right)$. Each recipient can now, with the help of the $k+j$ broadcast points plus his secret pseudoshare, reconstruct $p(x)$ and compute $s=p(0)$.

If the group of recipients changes, the polynomial scheme can be completely broken by the recipients in the intersection of both groups: If the group of recipients changes, e.g. w.l.o.g. from $\mathcal{U}=\left\{U_{1}, \ldots, U_{h}, U_{h+1}, \ldots, U_{k}\right\}$ to $\mathcal{U}^{\prime}=\left\{U_{1}, \ldots, U_{h}, U_{h+1}^{\prime}, \ldots, U_{l}^{\prime}\right\}$, then the polynomials for $\mathcal{U}$ and $\mathcal{U}^{\prime}$ intersect in those points which belong to the users $\left\{U_{1}, \ldots, U_{h}\right\}$. Each of these users can construct both polynomials, so each of them knows the secret pseudoshares of the $h-1$ other users.

The Vector Scheme. In the vector based scheme proposed in [Ber91], each user gets a pseudoshare $\left(V_{i}, y_{i}\right)$, where all the vectors $V_{i}=\left(v_{i 1}, \ldots, v_{i n}\right) \in G F(q)^{n}$ form an independent set. To broadcast a secret $s$, the transmitter proceeds as follows:

- Choose random numbers $r_{j}$ for all non-recipients $U_{j}$.

- Compute a (random) vector $P=\left(p_{1}, \ldots, p_{n}\right)$ satisfying

- $P \cdot V_{i}=y_{i}$ for all recipients $U_{i}$, and

- $P \cdot V_{j}=r_{j}$ for all non-recipients $U_{j}$.

- Pick a random vector $U \in G F(q)^{n}$ with $U \cdot V_{i} \neq 0$ for any recipient.

- Broadcast $U$ and $Q:=P+s U$.

To compute the secret $s$, a recipient $U_{i}$ has to solve the equation $(Q-t U)$. $V_{i}=y_{i}$ for $t$, and he will get $t=s$. I.e. he has to compute

$$
s:=\left(Q \cdot V_{i}-y_{i}\right) \cdot\left(U \cdot V_{i}\right)^{-1} .
$$

After one round of the vector scheme, each user knows the vectors $U$ and $Q$. Each recipient can compute $s$ and therefore knows the vector $P=Q-s U$. So after each round, each of the recipients has learned, for any other recipient $U_{i}$, a linear equation

$$
p_{1} v_{i 1}+\ldots+p_{n} v_{i n}=y_{i}
$$

in the unknowns $v_{i 1}, \ldots, v_{i n}, y_{i}$. If any user can collect $n+1$ of these linear equations, he can compute the secret pseudoshare $V_{i}, y_{i}$ of $U_{i}$. This may for example be the case for user $U_{j}$ if $U_{j}$ and $U_{i}$ are both recipients in at least $n+1$ rounds of the vector scheme. 


\section{A PROVABLY SECURE BROADCASTING SCHEME}

From the attacks described in Section 2. we have learned that the pseudoshares used by the recipients should only be used once to construct a $(k, n)$ Threshold Scheme. We will therefore use, as described in [LY93], the output of a randomized one-way function $f(\cdot, \cdot)$ as pseudoshares. The two inputs to this one-way function are a random number $r$ which is needed for randomization, and the personal key $k_{i}$ of user $U_{i}$. We assume that each randomized instance $f(r, \cdot)$ of $f$ is a one-way function.

Definition 3 A function $h: X \rightarrow Y$ is called one-way function if $h(x)$ is easy to compute for all $x \in X$, but for virtually all elements of $y \in \operatorname{Im}(h)$ it is computationally infeasible to find any $x \in X$ such that $h(x)=y$.

A function $f: V \times W \rightarrow Y$ is one-way on both arguments if, for any $v \in V$ and $w \in W$, both functions $f(v, \cdot)$ and $f(\cdot, w)$ are one-way-functions.

Such a function $f$ can be constructed by composing a one-way function $h(\cdot)$ with an arbitrary surjective two-argument function $g(\cdot, \cdot)$ :

Lemma 1 Let $h: X \rightarrow Y$ be a one-way function, and let $g: V \times W \rightarrow X$ be an arbitrary function which is surjective on both arguments. Then $f(\cdot, \cdot):=$ $h(g(\cdot, \cdot))$ is a one-way function on both arguments.

Proof. Assume w.l.o.g. that $f(v, \cdot)$ is not a one-way function for some $v \in V$. Then for any $y$ in the image of $f(v, \cdot)$ we may compute with nonnegligible probability a preimage $(v, x)$, i.e. $f(v, x)=h(g(v, x))=y$. Then $x^{\prime}:=g(v, x)$ is a preimage of the one-way function $h$ for $y$, a contradiction.

In the following protocol we omit the dummy shares used in [Ber91] for the randomization of the scheme. In our model, randomization is introduced by the use of the random numbers $r$.

\section{Broadcast Protocol}

To broadcast a secret $s$ selectively to the recipient subset $\left\{U_{1}, \ldots, U_{k}\right\}$ of all users, the transmitter performs the following steps:

- The transmitter chooses a random number $r$.

- For $i=1, \ldots, k$ he computes the temporary pseudoshares

$$
s_{i}:=f\left(r, k_{i}\right) .
$$

- From $s$ and $s_{1}, \ldots, s_{k}$ the transmitter constructs a $(k+1, n)$-Threshold Scheme with $n \geq 2 k+1$. 
- The transmitter selects $k$ shares $t_{1}, \ldots, t_{k}$ different from the pseudoshares of the recipients from the $(k+1, n)$-Threshold Scheme.

- The transmitter broadcasts $r, t_{1}, \ldots, t_{k}$.

To compute the secret $s$, recipient $U_{i}$ has to perform the following steps:

- $U_{i}$ computes his pseudoshare $s_{i}=f\left(r, k_{i}\right)$.

- Using the $(k+1, n)$-Threshold Scheme and the $k+1$ shares $s_{i}, t_{1}, \ldots, t_{k}$ he reconstructs $s$.

Theorem 1 If one-way functions exist, the broadcast protocol is secure for one round, i.e. no coalition of users can compute the personal key of another user after executing one round of the protocol.

Proof. According to the definition of Threshold Schemes, non-recipients do not learn anything during one round of the protocol. Since they cannot help in an attack, they are omitted from our discussion. The strongest coalition of recipients who cooperate to get the secret key of a single user $U_{1}$ is therefore the group of attackers $U_{2}, \ldots, U_{k}$.

Theoretically, the attackers should not know anything about the temporary pseudoshare $s_{1}$ of $U_{1}$ after one round. However, there are special cases where the attackers could get information about $s_{1}$ :

- If a small $n$ is chosen in the $(k+1, n)$-Threshold Scheme (e.g. $n=$ $2 k+1)$, the attackers may simply carry out in parallel the attack described below, i.e. for each of the possible values of $s_{1}$. This can be avoided by choosing Threshold Schemes with large $n$, e.g. using polynomials over the finite field $G F(n)$.

- If some side information is known, the attackers could be able to compute $s_{1}$. E.g. in the polynomial scheme, if the $x$-coordinate of each participant is fixed, the attackers could learn the $y$-coordinate from the polynomial the can construct.

Therefore we assume that the recipients $U_{2}, \ldots, U_{k}$ know the pseudoshare $s_{1}$ of $U_{1}$ and that with this information they are able to compute the personal key $k_{1}$ of recipient $U_{1}$. This is the strongest imaginable attack for one round of the protocol. We can reformulate the fact that this attack is succesful in oracle notation: There is an oracle $\mathcal{O}$ such that, on input $s_{1}, k_{2}, k_{3}, \ldots, k_{k}, r, t_{1}, \ldots, t_{k}$ outputs the personal key $k_{1}$ :

$$
\mathcal{O}\left(s_{1}, k_{2}, k_{3}, \ldots, k_{k}, r, t_{1}, \ldots, t_{k}\right)=k_{1} .
$$

We can now use this oracle to invert the one-way function $f(r, \cdot)$, i.e. if we know $r$, then for any $y$ in the image of $f(r, \cdot)$ we can use the oracle to find 
an $x$ such that $y=f(r, x)$. To do this, we choose $x_{2}, x_{3}, \ldots, x_{k}$ at random and compute for $i=2, \ldots, k$ the values $y_{i}=f\left(r, x_{i}\right)$. Then we construct a $(k+1, n)$-Threshold Scheme using the shares $y, y_{2}, \ldots, y_{k}$ and a randomly chosen secret $s$. Finally the oracle computes

$$
\mathcal{O}\left(y, x_{2}, x_{3}, \ldots, x_{k}, r, t_{1}, \ldots, t_{k}\right)=x .
$$

We have shown that even the strongest imaginable attack results in a contradiction, namely that the one-way function $f(r, \cdot)$ is invertible. Therefore if one-way functions exist, it is impossible to break the broadcast protocol after one round.

We now have to show that it is also impossible to break the protocol after several rounds. Unfortunately the technique used in theorem 1 is not applicable in the case of multiple rounds. Heuristically, since in each round of the protocol another random one-way-function is used for the computation of the pseudoshares, knowing a sequence of pseudoshares should not compromise the secret personal key of $U_{1}$. Furthermore, since the attackers do not know the actual pseudoshare of $U_{1}$, the parallel attack on all possible pseudoshares will become infeasible because the number of all possible combinations increases exponentially with the number of rounds. We can turn these heuristic arguments into a proof if we use stronger assumptions on the one-way function $f$.

Theorem 2 Let $E(\cdot, \cdot)$ be a symmetric encryption algorithm which is resistant against known plaintext attacks [MOV97]. If $E(k, m)$ denotes the ciphertext where the message $m$ is encrypted under the key $k$, we can define the function $f$ used in the broadcast protocol as

$$
f(r, k):=E(k, r) .
$$

Then the broadcast protocol can not be completely broken, even after multiple rounds.

Proof. Assume on the contrary that the broadcast protocol could be broken after $d$ rounds. Then there is an oracle which, on input $s_{1}^{(1)}, \ldots, s_{1}^{(d)}, r^{(1)}, \ldots, r^{(d)}$, $k_{2}, \ldots, k_{m}, \mathbf{t}^{(1)}, \ldots, \mathbf{t}^{(d)}$ (where $\mathbf{t}^{(j)}:=\left\{t_{1}^{(j)}, \ldots, t_{k_{j}}^{(j)}\right\}$ denotes the shares broadcast in round $j$, and $m$ is the number of all users) outputs $k_{1}$ :

$$
\mathcal{O}\left(s_{1}^{(1)}, \ldots, s_{1}^{(d)}, r^{(1)}, \ldots, r^{(d)}, k_{2}, \ldots, k_{m}, \mathbf{t}^{(1)}, \ldots, \mathbf{t}^{(d)}\right)=k_{1} .
$$

This oracle can then be used in a known plaintext attack against the encryption algorithm $E(\cdot, \cdot)$ : An attacker first observes plaintexts $m_{1}, \ldots, m_{d}$ and the corrsponding ciphertexts $c_{1}, \ldots, c_{d}$. Then he encrypts the same plaintexts under $m-1$ randomly chosen keys $k_{2}, \ldots, k_{m}$. Finally he chooses $d$ secrets 
$s^{(1)}, \ldots, s^{(d)}$ and constructs, for $i=1, \ldots, d$, a Threshold Scheme through (the appropriate subset of) $s^{(i)}, c_{i}, E\left(k_{2}, m_{i}\right), \ldots, E\left(k_{m}, m_{i}\right)$. Then he uses all this as an input to the oracle $\mathcal{O}$ and gets

$$
\mathcal{O}\left(c_{1}, \ldots, c_{d}, m_{1}, \ldots, m_{d}, k_{2}, \ldots, k_{m}, \mathbf{t}^{(1)}, \ldots, \mathbf{t}^{(d)}\right)=k .
$$

This is a contradiction, because we have assumed that $E(\cdot, \cdot)$ is resistant against chosen plaintext attacks.

Remark. Theorem 2 does not guarantee information theoretic security. I.e. that the attackers learn "something" in each round. However, if the number of rounds is polynomially bounded in the length of the input, this knowledge is not sufficient to compute the secret.

To illustrate this remark, consider the following example: An attacker could monitor the random numbers $r$ transmitted during different rounds of the protocol. With some probability, the same number $r$ will be repeated after a certain number of rounds. (E.g. using the birthday paradox, this probability is roughly $1 / 2$ after $2^{|r| / 2}$ rounds.) If this occurs, if he was a participant in both rounds, and if the Polynomial Threshold scheme has been used, he can compute the intersection of the two polynomials and can thus compute the share $f\left(r, k_{i}\right)$ for all users $i$ who participated in both rounds.

Remark. Theorem 2 is also true for one-way functions which are resistant to known plaintext attacks. Such functions may be used in and would be sufficient for challenge-and-response protocols. We refer to encryption algorithms because there seems to be no open literature on these special one-way functions.

\section{KEY DISTRIBUTION WITHOUT SUBLIMINAL CHANNEL}

The main applications of the scheme presented in this paper may be seen in a regulatory context. In many countries, the use of encryption techniques is forbidden or subject to restrictions on the strength of the algorithm, i.e. its effective key length.

On the other hand, for certain services weak encryption algorithms are sufficient to protect the contents. But even if the encryption of the content itself may be weak, the key management itself should not. Therefore the broadcasting scheme presented in this paper could be used for key management, e.g. by succesively establishing a key hierarchy.

Another regulatory advantage of our scheme when compared to the separate encryption of session keys under the different personal keys is that in our scheme the regulatory authority can be sure that only one secret has been broadcast, and can efficiently check this secret.

To achieve both goals, namely to establish a strong key distribution scheme, and to guarantee that there is no subliminal channel, we proceed as follows: 
- The legal authority who is allowed to control the information flow in the scheme gets two personal keys $k_{0}$ and $k_{-1}$.

- The random number $r$ is derived from the secret $s$ via some one-way function $f: r:=f(s)$.

- For $i=-1,0,1, \ldots, k$ the transmitter computes the temporary pseudoshares

$$
s_{i}:=f\left(r, k_{i}\right) .
$$

- From $s$ and $s_{-1}, s_{0}, s_{1}, \ldots, s_{k}$ the transmitter constructs a $(k+3, n)$ Threshold Scheme with $n \geq 2 k+5$.

- The transmitter selects $k+2$ shares $t_{1}, \ldots, t_{k+2}$ different from the pseudoshares of the recipients from the $(k+3, n)$-Threshold Scheme.

- The transmitter broadcasts $r, t_{1}, \ldots, t_{k+2}$.

To check if no secret message has been transmitted, the legal authority proceeds as follows:

- The legal authority computes its pseudoshares $s_{-1}=f\left(r, k_{-1}\right)$ and $s_{0}=f\left(r, k_{0}\right)$.

- Using the $(k+3, n)$-Threshold Scheme and the $k+4$ shares known by him the legal authority checks if the pseudoshares $s_{-1}, t_{1}, \ldots, t_{k+2}$ and $s_{0}, t_{1}, \ldots, t_{k+2}$ reveal the same secret $s$.

- The authority checks if $r:=f(s)$.

The transmitter cannot fool the authority by not taking into account the temporary pseudoshares of the authority in the construction of the $(k+3, n)$ Threshold Scheme, since in this case the probability that the two shares of the authority give the same secret is negligible. There is also no chance to hide some secret information $m$ in the random number $r$ and to transmit it to one recipient $U_{i}$ (e.g. by choosing $r:=E\left(k_{i}, m\right)$ ) since $r$ depends in a well-known manner on the value $s$ known by the authority.

\section{APPLICATIONS}

One special application can be found in the area of Pay-TV systems. Analogue Pay-TV systems use relatively weak encryption algorithms for their audio/video contents, and weak encryption will also be sufficient for digital systems because the content only has a temporary value and therefore only has to be protected for some (short) time. E.g. a football match sold as a Pay-TV event is worthless as soon as the game is over, so a weak encryption algorithm 
would be sufficient to protect the content against an exhaustive search attack for roughly two hours.

On the other hand, the entitlements to watch certain films or channels have to be distributed in a very secure manner via a broadcast channel. Most Pay-TV systems solve this problem by giving a personal key to each user stored in a secure environment (e.g. a smart card), and then broadcasting cryptograms containing the entitlements ciphered with the personal keys. (For efficiency reasons, group keys are introduced so that an entitlement can be sent to a group of users.)

Now applying e.g. US export restrictions to systems which distribute entitlements would create a major weakness in these systems, because entitlements have a validity of at least one month. On the other hand an exhaustive search attack on a cryptogram ciphered with a 40 bit key will take only some hours, especially when a kind of Chinese lottery attack can be used. Even 56 Bit keys are now within the reach of exhaustive search attacks, as has been demonstrated by the Electronic Frontier Foundation on January 19th, 1999 [EFF99].

Using our scheme to distribute entitlements can solve these problems since it can be used since there are arbitrarily strong symmetric encryption algorithms which are secure against chosen plaintext attacks, and so the security of the overall system is guaranteed by Theorem 2 .

\section{OPEN POINTS}

It is not clear if attacks simlar to those described in Section 2. exist for the general scheme described in [Ber91] since our attacks make use of the algebraic properties of special implementations.

It would be interesting to characterize one-way functions which are resistant to known plaintext attacks since they could provide a faster alternative to the use of symmetric encryption functions in challenge-and-response protocols. In addition, by using such functions the features of authentication and encryption could be better separated to facilitate export of strong authentication.

\section{References}

[BC94] C. Blundo and A. Cresti, Space Requirements for Broadcast Encryption. Proc. EUROCRYPT '94, Springer LNCS 950, pp. 287-298.

[Ber91] S. Berkovits, How To Broadcast A Secret. Proc. EUROCRYPT '91, Springer LNCS 547, pp. 536-541.

[EFF99] Cracking DES. Electronic Frontier Foundation. http://www.eff.org/descracker/.

[FN93] A. Fiat and M. Naor, Broadcast Encryption. Proc. CRYPTO '93, Springer LNCS 773, pp. 480-491. 
[JKKO95] M. Just, E. Kranakis, D. Krizanc and P. van Oorschot, On Key Distribution via True Broadcasting. Proc. 2nd ACM Conf. on Computer and Communications Security (1995), pp. 81-88.

[LS98] M. Luby and J. Staddon, Combinatorial Bounds for Broadcast Encryption. Proc. EUROCRYPT '98, Springer LNCS 1403.

[LY93] Chi-Sung Laih and Sung-Ming Yen, On the Design of Conference Key Distribution Systems for the Broadcasting Networks. Proc. IEEE Infocom '93, IEEE Press Washington, pp. 1406-1413.

[MOV97] A. J. Menezes, P. C. van Oorschot and S. A. Vanstone, Handbook of Applied Cryptography. CRC Press, New York 1997.

[Sha79] A. Shamir, How to Share a Secret. Comm. ACM, Vol. 22, No. 11, November 1979, pp. 612-613.

[Sti97] D. R. Stinson, On some methods for unconditionally secure key distribution and broadcast encryption. Designs, Codes and Cryptography, 12 (1997), 215-243.

[SvT98] D. R. Stinson and T. van Trung, Some new results on key distribution patterns and broadcast encryption. Designs, Codes and Cryptography, 14 (1998), 261-279. 